\title{
REVIEW
}

\section{Group B streptococcal conjugate vaccines}

\author{
C J Baker, M S Edwards
}

Arch Dis Child 2003;88:375-378

Linkage of bacterial capsular polysaccharides to proteins to create conjugate vaccines has had a dramatic impact on the health of children. Although unconjugated polysaccharides are poorly immunogenic in infants and some older children and adults, their covalent coupling with proteins stimulates $T$ cell dependent antigenic recognition that profoundly enhances immunogenicity. In the decade since the introduction and widespread use of Haemophilus influenzae type $b$ polysaccharide conjugate vaccines in the United States, invasive $H$ influenzae infections have become a rarity in childhood. 'Similarly, the conjugation of polysaccharides of Streptococcus pneumoniae to a derivative of diphtheria toxoid and the addition of pneumococcal conjugate vaccine to infant immunisation schedules carries with it promise for a similar decline in the incidence of invasive pneumococcal disease in paediatric patients. ${ }^{2}$

See end of article for authors' affiliations

Correspondence to:

Dr M S Edwards, Baylor

College of Medicine, One Baylor Plaza, Room 302A,

Houston, Texas 77030,

USA; morvene@

bcm.tmc.edu

Accepted

5 October 2002 nvasive disease caused by group B streptococcus (GBS) remains a major cause of mortality and morbidity in neonates and young infants. Despite the introduction and widespread implementation of maternal intrapartum antibiotic prophylaxis (IAP) in the USA since 1996, an estimated 2200 neonates during the first week of life and 1400 infants 7-89 days of age will develop invasive GBS disease annually; 140 of these cases will be fatal. ${ }^{3}$ While maternal IAP is unquestionably effective and has reduced the incidence of early onset disease by nearly $75 \%$, it is at best an interim intervention that does not prevent all early onset and has no influence on the incidence of late onset GBS disease. For example, since $20-25 \%$ of delivering women in the USA currently are receiving IAP, the development of penicillin resistance among GBS isolates is a concern that theoretically could render this intervention ineffective. Already a recent and substantial increase in resistance to erythromycin and clindamycin among GBS has been documented by several groups of investigators. ${ }^{4-6}$

The intent of this review is to provide an update on the status of GBS polysaccharide-protein conjugate vaccines. The review will present the rationale for the development of GBS conjugate vaccines, the current status of clinical trials with these vaccines, and prospects for the future use of GBS conjugate vaccines.

\section{THE RATIONALE FOR GBS POLYSACCHARIDE-PROTEIN CONJUGATE VACCINES}

A protective role for antibodies to the capsular polysaccharide (CPS) of type III GBS was predicted in the 1970s by Baker and Kasper. ${ }^{7}$ These investigators showed that infants with invasive early or late onset GBS disease were born to mothers with very low levels of III CPS specific antibodies in sera at delivery. The finding that human sera containing a sufficient concentration of CPS specific IgG promoted efficient opsonisation and phagocytosis in vitro and provided protection from lethal experimental infection affirmed the importance of capsular serotype specific antibodies. This information, combined with that showing the efficacy of pneumococcal polysaccharide vaccine in adults, suggested that active immunisation of pregnant women with purified GBS polysaccharides could protect neonates and young infants. The premise was that if vaccination elicited protective levels of antibodies in childbearing age women, placental passage of these GBS CPS specific antibodies would protect neonates throughout the period of disease risk for invasive GBS infection (for example, the first 2-3 months of life).

Once the GBS three major serotypes that caused invasive GBS disease (Ia, II, and III) were structurally and immunochemically defined, there was a sense of expectation among investigators in the field. The first candidate GBS vaccine, a purified type III CPS, underwent phase 1 testing in healthy adults in 1978; subsequently type Ia and II CPS vaccines were studied. By contrast to the excellent immunogenicity observed in adults immunised with multivalent pneumococcal polysaccharide vaccine, type Ia, II, and III polysaccharides, while well tolerated, had uneven immunogenicity. Most adults (nearly 90\%), it was discovered, had very low preimmunisation serum concentrations of CPS specific antibodies. These low levels, presumably indicating immunologic naivety to GBS polysaccharides, predicted a poor immune response in many, so that only $40 \%$ and $60 \%$, respectively, developed significant increases in specific antibodies after immunisation with type Ia and III polysaccharides. By contrast, $88 \%$ of those immunised with type II CPS responded. ${ }^{8}$ In the few adults who presumably had been "primed" to GBS polysaccharides or related antigens (indicated by moderately increased serum

Abbreviations: CPS, capsular polysaccharide; GBS, group B streptococcus; GMC, geometric mean concentration; IAP, intrapartum antibiotic prophylaxis; $T T$, tetanus toxoid 
Table 1 Group B streptococcal CPS-TT conjugate vaccines

\begin{tabular}{|c|c|c|c|c|}
\hline GBS vaccine & $\begin{array}{l}\text { Approximate CPS } \\
\text { dose }(\mu \mathrm{g} / \mathrm{ml})\end{array}$ & $\begin{array}{l}\text { Proportion of adults with } \\
\text { greater than fourfold } \\
\text { rises in CPS specific IgG } \\
\text { at } 8 \text { weeks }\end{array}$ & $\begin{array}{l}\text { GMC }(\mu \mathrm{g} / \mathrm{ml}) \text { of CPS } \\
\text { specific lgG }(95 \% \mathrm{Cl}) \\
\text { at } 8 \text { weeks }\end{array}$ & $\begin{array}{l}\text { Estimated } \\
\text { percent of } \\
\text { infant disease } \\
\text { in the USA by } \\
\text { GBS serotype }\end{array}$ \\
\hline la-TT* & 15 & $80 \%$ & $18.3(6.0$ to 55.4$)$ & 25 \\
\hline $\mathrm{lb}-\mathrm{TT}$ * & 16 & $80 \%$ & 11.1 (3.3 to 37.0$)$ & 5 \\
\hline$\|-T^{*}$ & 4 & $87 \%$ & $11.2(6.1$ to 20.8$)$ & 5 \\
\hline III-TT* & 13 & $93 \%$ & $2.7(1.0$ to 7.8$)$ & 50 \\
\hline $\mathrm{V}-\mathrm{TT}^{*}$ & 10 & $93 \%$ & 5.5 (1.5 to 20.0 ) & 15 \\
\hline
\end{tabular}

*Data from reference 14 (la and Ib), 15 (II), and 16 and 17 (III). Unpublished observations for type V.

†Data from references 10 and 11 .

concentrations of CPS specific antibodies), immunisation with type Ia, II, and III polysaccharides elicited brisk immune responses in nearly $100 \%$.

The trials with GBS polysaccharide vaccines verified the feasibility of immunisation as an approach to prevent GBS disease and revealed the need to develop candidate vaccines with improved immunogencity. One encouragement to the ultimate success of a GBS vaccine programme was the first study conducted in pregnant women. Among responders to a type III GBS CPS vaccine administered at about 31 weeks of gestation, $90 \%$ had infants with substantial levels of III CPS specific antibodies in cord sera that promoted opsonisation and killing of type III GBS in vitro; this functional activity of infant sera persisted in the majority until age 3 months. Thus, in concept, maternal immunisation with an optimally immunogenic GBS vaccine during the third trimester should protect neonates and young infants from invasive GBS infection during the age of risk.

\section{THE CURRENT STATUS OF GBS CONJUGATE VACCINES}

In the early 1990s a new GBS serotype, type V, emerged among cases in the USA and in other countries. A recent multicity active surveillance study of invasive GBS disease performed in a racially and ethnically diverse cohort of pregnant women and neonates noted that type $\mathrm{V}$ accounted for $23 \%$ and $14 \%$, respectively, of maternal and early onset neonatal cases. ${ }^{10}$ The emergence of type $\mathrm{V}$ and an increasing number of nontypeable GBS isolates from colonised adults underscores the importance of ongoing epidemiologic investigations in formulating an appropriate multivalent GBS vaccine. Taken together, types Ia, III, and V GBS now account for at least $75 \%$, and five serotypes (Ia, Ib, II, III, and V) for virtually $100 \%$ of GBS disease in infants and adults. ${ }^{11}$

Development of the first GBS conjugate vaccine, type III polysaccharide-tetanus toxoid, was driven by its prominence among infant isolates, especially those with meningitis and late onset infection with any clinical presentation. This type III CPS was covalently linked to monomeric tetanus toxoid using reductive amination coupling chemistry. ${ }^{12}$ All GBS conjugates to date were produced with this coupling chemistry, and with one exception, all studies to date have evaluated conjugates in healthy adults using tetanus toxoid as the protein carrier.

In the first clinical evaluation of a GBS conjugate, 100 women aged 18-40 years were randomised to receive either type III CPS-tetanus toxoid (III-TT) conjugate vaccine at one of three dosages, uncoupled III CPS, or placebo (saline).${ }^{13}$ Each vaccine was well tolerated, and the predominant III CPS specific antibody isotype ( $>95 \%$ ) elicited by immunisation was IgG. Although higher geometric mean CPS specific IgG levels were achieved in response to higher dosages of the conjugate, greater than fourfold rises were achieved in $90 \%$ of recipients of III-TT but in only $50 \%$ III CPS recipients $(p=0.0015)$. The III CPS specific IgG elicited by immunisation was functional in vitro and in protected neonatal mice from a lethal type III GBS challenge. Thus conjugation to TT significantly enhanced immunogenicity and indicated that prevention of perinatal GBS disease through maternal immunisation was potentially feasible.

Since 1996, conjugate vaccines to each of the clinically significant GBS serotypes causing invasive disease have been developed and tested in nearly 500 healthy young adults. ${ }^{14-17}$ Each of these vaccines has been well tolerated when given as a single intramuscular dose or as two doses. No serious vaccine associated events have occurred. Most recipients have had only mild to moderate tenderness at the injection site or no discernable symptoms or signs. Systemic reactions, consisting of low grade fever, chills, headache, or myalgias that resolved within 24-36 hours occurred in less than $2 \%$ of nearly 500 volunteers.

Dose-response testing has been performed for Ia, Ib, II, III, and V CPS-TT conjugates to determine the minimum dose that could be used in a multivalent vaccine (table 1). Such data are necessary to minimise the total amount of polysaccharide needed to stimulate a protective level of immunity and the rate of local reactions that appear to depend primarily on total tetanus carrier dose. Immune responses to GBS conjugates, with the exception of type $\mathrm{V}$, are dose dependent. Doses of $\sim 4$ $\mu \mathrm{g}$ for type II CPS, $10 \mu \mathrm{g}$ for type $\mathrm{V}$, and $\sim 15 \mu \mathrm{g}$ for types Ia, Ib, and III CPS have elicited greater than fourfold increases in CPS specific IgG in $80-93 \%$ of volunteers eight weeks post-immunisation. For each serotype, conjugation has enhanced significantly immunogenicity when compared to uncoupled polysaccharides. Vaccine responses generally reach a peak four to eight weeks after immunisation and then decline. At one year after immunisation, the geometric mean concentration (GMC) of antibody is approximately $50 \%$ of the peak level. For example, subjects immunised with a $15 \mu \mathrm{g}$ dose of type Ia CPS-TT have a GMC of $18.3 \mu \mathrm{g} / \mathrm{ml}$ at eight weeks after immunisation and $9.1 \mu \mathrm{g} / \mathrm{ml}$ at one year. ${ }^{14}$ Based on limited data at two years after immunisation, the GMC is still well above baseline, suggesting that as with other conjugate vaccines, the response to GBS conjugate vaccines is durable (Baker and colleagues ${ }^{14}{ }^{15}$; also Baker et al, unpublished observations).

Functional activity has been tested in vitro with an opsonophagocytosis assay. For each serotype, positive correlations exist between killing of homologous GBS strains by human neutrophils after opsonisation by complement and concentration of conjugate vaccine induced CPS specific IgG. ${ }^{13-16}$ These findings suggest that GBS conjugates of a design similar to those already tested will be effective in GBS disease prevention. Table 1 summarises the immune responses to theoretically optimal dosages of each GBS conjugate vaccine tested in healthy adults and the proportion of disease in pregnant women and young infants (early and late onset disease) caused by the five serotypes. The magnitude of immune responses suggests that a single dose of a pentavalent GBS conjugate vaccine might be expected to have an efficacy of nearly $90 \%$. 
With a pentavalent vaccine as the goal, preliminary evaluation of a combination of types II-TT and III-TT vaccines has been conducted. ${ }^{17}$ This bivalent II/III CPS-TT conjugate vaccine was well tolerated in healthy adults and no immune interference was noted when compared to vaccinees who received a monovalent II-TT or III-TT conjugate. The bivalent vaccine stimulated immune responses comparable to those elicited by either of the monovalent conjugates. Another question of importance is whether a single dose of GBS conjugate vaccine will elicit an optimal response among a majority of recipients or whether a "booster" will be required. To address this, a group of healthy adults were given a second dose of III-TT vaccine 21 months after their initial immunisation. ${ }^{16}$ No increase in reactogenicity was observed, but the GMC of III CPS specific IgG eight weeks after the second dose was similar to that noted after the initial dose, indicating no discernable booster effect for the group as a whole. A small proportion of adults, some of these with the lowest preimmunisation antibody concentrations, did exhibit a "booster" response. Further studies will be required to prove the hypothesis that these individuals were truly naïve to the antigen, were "primed" with the first dose, and subsequently had an optimal response.

Two lots of type $\mathrm{V}$ conjugate vaccine have been tested in phase 1 clinical trials (Baker CJ, unpublished observations). The first used TT and the second the mutant diphtheria toxin, cross reactive material $197\left(\mathrm{CRM}_{197}\right)$. The two conjugates were similarly well tolerated. Type V-TT recipients had slightly higher CPS specific IgG levels in their sera after immunisation than the V-CRM ${ }_{197}$ group, but the differences were not significant. These findings, using $\mathrm{CRM}_{197}$ as a protein carrier, are important because they affirm that more than one carrier can recruit the $\mathrm{T}$ cell help needed for an adequate immune response to a GBS polysaccharide.

As with the uncoupled GBS polysaccharide, a phase 1 trial in women at 32-34 weeks of gestation has just been completed. ${ }^{18}$ After randomisation, III-TT conjugate (12.5 $\mu \mathrm{g}$ dose of III CPS) or saline placebo was administered to 20 and 10 women, respectively. III CPS specific IgG concentrations were determined in maternal sera at delivery (at a mean of 8.3 weeks after immunisation), infant cord sera, and in sera obtained from infants at 1 and 2 months of age. GMCs in delivery sera from immunised women were significantly increased from preimmunisation values and correlated well $(r=0.93)$ with infant cord values. The actual GMCs in sera from infants of vaccinated women were 3.7 and $2.2 \mu \mathrm{g} / \mathrm{ml}$, respectively, at 1 and 2 months of age. Sera from these infants of vaccinated women uniformly promoted opsonisation of type III GBS and killing by neutrophils in vitro. Thus, conjugate vaccine induced III CPS specific IgG was efficiently transported to the neonate and functions well in vitro, suggesting that a multivalent GBS conjugate vaccine has the potential for prevention of late as well as of early onset infant GBS disease, and possibly to effect a decrease in maternal invasive disease also.

\section{BARRIERS TO LICENSURE OF GBS CONJUGATE VACCINES}

Over two decades of investigation verifies the notion that maternal immunisation with a multivalent GBS conjugate vaccine is a valid approach to prevention of GBS disease in infancy. Published data indicate that candidate GBS polysaccharide-tetanus toxoid conjugates are safe in adults and elicit antibodies well above what is likely to be passively protective for neonates and young infants. ${ }^{19}{ }^{20}$ The remaining scientific issue that must be answered for licensure is documentation of the efficacy of a GBS conjugate vaccine in a phase 3 trial. In order to facilitate such a trial, the formation of a corporate partnership with a pharmaceutical company would be desirable. There are concerns regarding such an undertaking. One obvious roadblock is a longstanding hesitancy to confront liability issues that surround immunisation of pregnant women. However, millions of doses of tetanus toxoid have been given to pregnant women without adverse effects. ${ }^{21}$ Since the GBS polysaccharides are simple sugars, it is difficult to conceive of their having other than a salutary effect on pregnant women and their fetuses when administered early in the third trimester. It has been suggested that an injury compensation programme, similar to those in effect for childhood immunisations, should be developed for maternal immunisation programmes to create an environment conducive for providers and encouraging to the vaccine industry. ${ }^{22}$ The formation, in the 1990s, of such public advocacy groups as the Group B Strep Association in the United States and the Canadian Group B Strep Association in Canada have fostered media attention on the importance of making vaccine prevention of GBS infection a reality. ${ }^{23}$ While other target populations, such as nonpregnant, childbearing age women and even adolescents, could be considered for immunisation, efficacy testing would not be practical in such populations. However, nonpregnant adults with defined medical conditions such as diabetes mellitus, chronic liver disease, and malignancy, or those more than 65 years of age would be reasonable target populations given their current invasive GBS disease burden. ${ }^{24-26}$ To date, almost no information exists regarding potentially protective CPS specific antibodies in these patients, whether other immune defence abnormalities render protective levels functionally inadequate, and if GBS conjugate are adequately immunogenic. The scientific evidence for maternal immunisation to prevent perinatal GBS disease is compelling. If perinatal GBS disease is to become, as with $H$ influenzae type b disease in infants, a rarity, the time for pharmaceutical industry leadership is now.

\section{Authors' affiliations}

C J Baker, M S Edwards, Section of Infectious Diseases, Department of Pediatrics, Baylor College of Medicine, Houston, Texas 77030, USA

\section{REFERENCES}

1 Centers for Disease Control and Prevention. Progress toward eliminating Hemophilus influenzae type $b$ disease among infants and children-United States, 1987-1997. Morbid Mortal Weekly Rep 1998;47:993-8.

2 Obaro S, Adegbola R. The pneumococcus: carriage, disease and conjugate vaccines. J Med Microbiol 2002;51:98-104.

3 Schrag SJ, Zywicki S, Farley MM, et al. Group B streptococcal disease in the era of intrapartum antibiotic prophylaxis. N Engl J Med 2000;342: 15-20.

4 Fernandez $M$, Hickman ME, Baker CJ. Antimicrobial susceptibilities of group B streptococci isolated between 1992 and 1996 from patients with bacteremia or meningitis. Antimicrob Agent Chemother 1998;42:1517-19.

5 Pearlman MD, Pierson CL, Faix RG. Frequent resistance of clinical group B streptococci isolates to clindamycin and erythromycin. Obstet Gynecol 1998;92:258-61.

6 Murdoch DR, Reller LB. Antimicrobial susceptibilities of group B streptococci isolated from patients with invasive disease: 10-year perspective. Antimicrob Agent Chemother 2001;45:3623-4.

7 Baker CJ, Kasper DL. Correlation of maternal antibody deficiency with susceptibility to neonatal group B streptococcal infection. N EnglJ Med 1976;294:753-6.

8 Baker CJ, Kasper DL. Group B streptococcal vaccines. Rev Infect Dis 1985; 7:458-67.

9 Baker CJ, Rench MA, Edwards MS, et al. Immunization of pregnant women with a polysaccharide vaccine of group B streptococcus. N Engl J Med 1988;319:1180-5.

10 Zaleznik DF, Rench MA, Hillier S, et al. Invasive disease due to group B streptococcus in pregnant women and neonates from diverse population groups. Clin Infect Dis 1999:30:276-81.

11 Harrison LH, Elliott JA, Dwyer DM, et al. Serotype distribution of invasive group $B$ streptococcal isolates in Maryland: implications for vaccine formulation. J Infect Dis 1998;177:998-1002.

12 Wessels MR, Paoletti LC, Kasper DL, et al. Immunogenicity in animals of a polysaccharide-protein conjugate vaccine against type III group $B$ streptococcus. J Clin Invest 1990;86: 1428-33. 
13 Kasper DL, Paoletti LC, Wessels MR, et al. Immune response to type III group B streptococcal polysaccharide-tetanus toxoid conjugate vaccine. J Clin Invest 1996;98:2308-14

14 Baker CJ, Paoletti LC, Wessels MR, et al. Safety and immunogenicity of capsular polysaccharide-tetanus toxoid conjugate vaccines for group $B$ streptococcal types la and Ib. J Infect Dis 1999;179:142-50.

15 Baker CJ, Paoletti LC, Rench MA, et al. Use of capsular polysaccharide-tetanus toxoid conjugate vaccine for type II group $B$ streptococcus in healthy women. J Infect Dis 2000;182:1129-38.

16 Paoletti LC, Rench MA, Kasper DL, et al. Effects of alum adjuvant or a booster dose on immunogenicity during clinical trials of group $B$ streptococcal type III conjugate vaccines. Infect Immun 2001;69:6696-701

17 Fernandez M, Paoletti LC, Kasper DL, et al. Evaluation of a bivalent group $B$ streptococcal polysaccharide-tetanus toxoid conjugate vaccine [abstract 33]. Clin Infect Dis 1999;29:966.

18 Baker CJ, Rench MA, Mclnnes P. Safety and immunogenicity of group B streptococcal (GBS) type III capsular polysaccharide (CPS)-tetanus toxoid (III-TT) conjugate vaccine in pregnant women [abstract 370]. Clin Infect Dis 2001;33:1151
19 Lin F-YC, Philips III JB, Azimi PH, et al. Level of maternal antibody required to protect neonates against early-onset disease caused by group B streptococcus type la: a multicenter seroepidemiology study. J Infect Dis 2001; 184:1022-8.

20 Baker CJ, Carey VJ, Edwards MS, et al. Quantity of maternal antibody at delivery that protects neonates from early-onset group B streptococcal disease. JAMA, submitted.

21 Whitman C, Belgharbi L, Gasse F, et al. Progress towards the global elimination of neonatal tetanus. World Health Stat Q 1992;45:248-56.

22 Paradiso PR. Maternal immunization: the influence of liability issues on vaccine development. Vaccine 2002;20:S73-4.

23 Schuchat A. Group B streptococcal disease: from trials and tribulations to triumph and trepidation. Clin Infect Dis 2001;33:751-6.

24 Farley MM. Group B streptococcal disease in nonpregnant adults. Clin Infect Dis 2001;33:556-61.

25 Muñoz P, Llancaqueo A, Rodríguez-Créixems M, et al. Group B streptococcus bacteremia in nonpregnant adults. Arch Intern Med 1997; 157:213-16.

26 Henning KJ, Hall EL, Dwyer DM, et al. Invasive group B streptococcal disease in Maryland nursing home residents. J Infect Dis 2001;183:1138-42.

\section{IMAGES IN PAEDIATRICS}

\section{Trichobezoar}

A 13 year old adolescent girl presented with vague abdominal pain of a week's duration. The pain was not associated with vomiting, fever, or weight loss. She was thin built and had mild pallor. Examination of the abdomen revealed a large curvilinear hard intraabdominal mass, occupying the epigastric and left hypochondriac regions (fig 1). Abdominal skiagram and ultrasonography were suggestive of gastric bezoar. A history of pica and ingestion of hair strands was obtained subsequently. Laparotomy was performed and a huge trichobezoar was removed from the stomach in toto successfully (fig 2 ).

Bezoar is a concretion of material in the lumen of the digestive tract ingested over time. ${ }^{1}$ Four types of bezoar have been described: phytobezoar (vegetable material); trichobezoar (hair); lactobezoar; and miscellaneous. The presenting symptoms are abdominal pain, vomiting, early satiety, anorexia, weight loss, abdominal mass, and rarely gastric obstruction. Abdominal skiagram and upper gastrointestinal contrast study are diagnostic. Therapeutic options consist of endoscopic fragmentation and removal, and dissolution with substances such as papain, saline, acetylcysteine, and cellulase. Extracorporeal shock wave lithotripsy and laser therapy have been described as therapeutic modalities. ${ }^{2}$ Trichobezoars virtually always require surgical removal. ${ }^{3}$ They are often found in emotionally disturbed patients and warrant a psychiatric evaluation to prevent recurrence. ${ }^{2}$

B Sundaram, N Selvarajan, S Somu, A N Kamalanathan Kanchi Kamakoti CHILDS Trust Hospital, 12A Nageswara Road, Nungambakkam, Chennai, Ta, India; akamalanathan@rediffmail.com

\section{References}

1 Schreiber RA. Disorders of the stomach. In: Walker Smith JA, Hamilton R, Walker AW, eds. Practical pediatric gastroenterology, 2nd edn. Jaypee Brothers, 1996:154.

2 Sigalet DL, Snyder CL. Lesions of the stomach. In: Ashcraft KW, Murphy JP, Sharp $\mathrm{R}$, eds. Textbook of pediatric surgery, 3rd edn. W.B. Saunders Company, 2000:397-8. 3 Magnuson DK, Schwartz MZ. Stomach and duodenum. In: Oldham KT, Colombani PM, Foglia RP, eds. Surgery of infants and children-scientific principles and practice, $1 \mathrm{st}$ edn. Lippincott-Raven, 1997:1159.

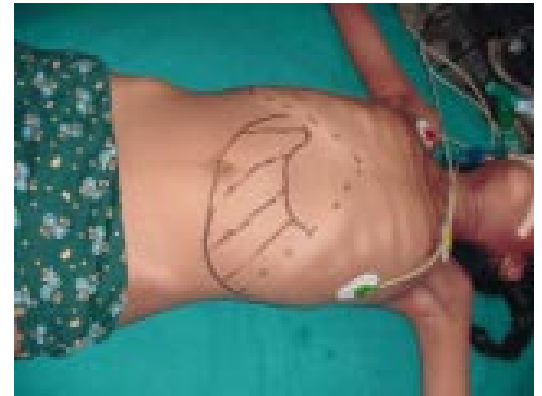

Figure 1

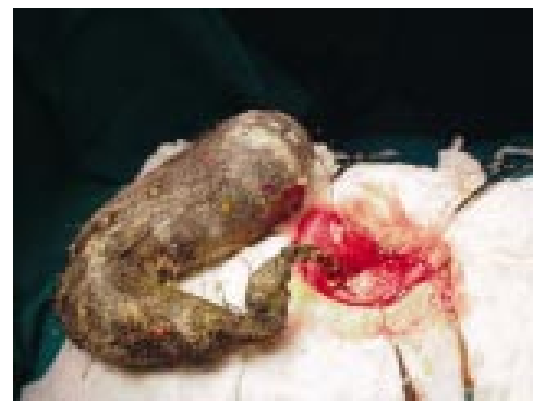

Figure 2 